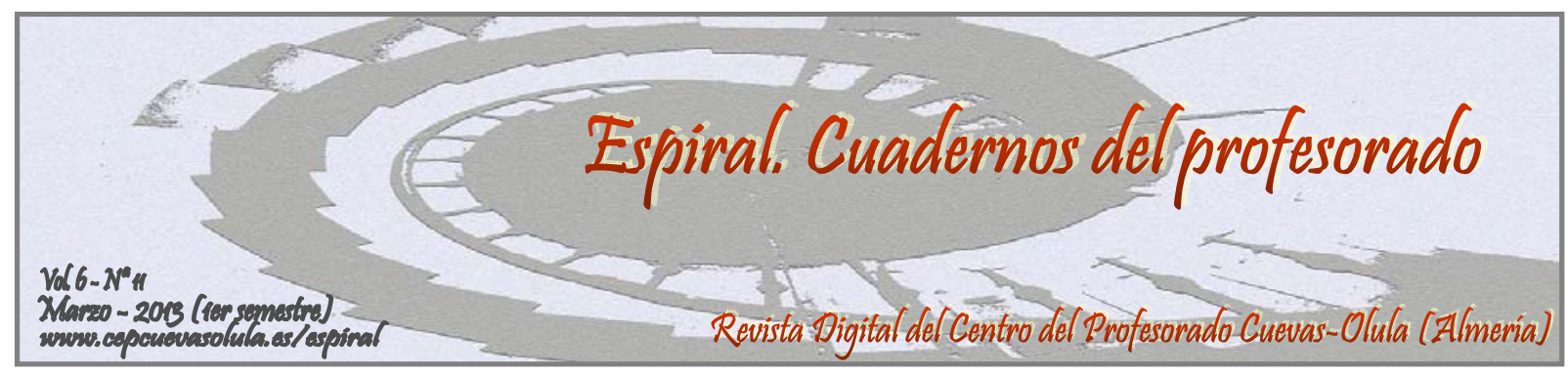

\title{
EL PIRAGÜISMO EN LA FORMACIÓN PERMANENTE DEL PROFESORADO COMO ACTUALIZACIÓN DE CONTENIDOS EN EDUCACIÓN FÍSICA
}

\section{CANOEING IN TEACHER CONTINOUS TRAINING AS UPDATE OF CONTENTS OF PHYSICAL EDUCATION Antonio Granero-Gallegos ${ }^{(1)}$ y Antonio Baena-Extremera ${ }^{(2)}$}

(1) Centro del Profesorado Cuevas-Olula, Junta de Andalucía, España

(2) Facultad de Ciencias del Deporte, Universidad de Murcia, España

RESUMEN: El profesorado que imparte su ejercicio profesional docente en centros no universitarios, una vez finalizada su formación inicial, necesita de la denominada formación permanente para actualizar sus conocimientos y sus métodos a la evolución científica y pedagógica. En el desarrollo del Plan de Actuación del Centro del Profesorado Cuevas-Olula se incardina esta actividad formativa que tiene como objetivo la actualización de contenidos del bloque de actividad física en el medio natural, como recoge el R.D. 1631/2006, concretados en el piragüismo, tratando de despertar el interés hacia esta práctica deportiva, proporcionando experiencias en distintos contenidos de aprendizaje, con el fin de formar al profesorado para que sea capaz de llevar al alumnado hacia el conocimiento del piragüismo y a la primera toma de contacto de la actividad. En este caso, además, se persigue la promoción y utilización con carácter docente del Canal de Remo de Cuevas del Almanzora, construido con motivo de los Juegos del Mediterráneo, celebrados en Almería en 2005. Las sesiones prácticas se han realizado combinando distintas características de entorno y aguas (puerto, playa y río), aspecto gran valorado al finalizar la actividad. Es destacable la alta valoración de este curso por parte de los participantes y el alto grado de consecución de los objetivos. Por último, resaltar que la celebración de esta actividad formativa ha tenido una repercusión muy positiva y ha supuesto un incentivo más para que a nivel municipal se tomaran las iniciativas oportunas y el Canal de Remo se pusiera en funcionamiento.

Palabras clave: formación permanente, profesorado, Educación Física, piragüismo.

ABSTRACT: Teachers who taught at non-university teaching centres, after their initial training, requires the so-called training to update their knowledge and methods to the scientific and educational developments. In developing the Action Plan of the Faculty Centre (EPC)-Cuevas Olula is to ground this educational activity which aims to update the contents of the block of physical activity on the natural environment, as reflected in the RD 1631/2006, particularly in canoeing, trying to arise interest in this sport, providing different experiences in learning contents in order to train teachers to be able to lead students to knowledge of canoeing and the first touchdown with the activity. In this case, pursuing also the promotion of the Rowing Channel of Cuevas del Almanzora, built during the Mediterranean Games held in Almeria in 2005 with an educational purpose. The practice sessions have been conducted by combining different features of environment and waters (harbour, beach and river), which is of great value at the end of the activity. It is worth the high rating of this course participants and the high degree of achievement of objectives. Finally, to emphasize the fact that this training activity, has had a very positive impact and has been a further incentive to heal for initiatives were taken on the part of Counsellor and Rowing Channel was in operation.

Key words: continuous training, teachers, physical education, canoeing. 
Granero-Gallegos, A., y Baena-Extremera, A. (2013). El piragüismo en la formación permanente del profesorado como actualización de contenidos en Educación Física. Espiral. Cuadernos del Profesorado, 6(11), 3-11. Disponible en: http://www.cepcuevasolula.es/espiral.

Fecha de recepción: 11/09/2012

Fecha de aceptación: 26/11/2012
Enviar correspondencia a: agranerog@hotmail.com

\section{1.- INTRODUCCIÓN}

El profesorado que imparte su ejercicio profesional docente en centros no universitarios, una vez finalizada su formación inicial, necesita de la denominada formación permanente para actualizar sus conocimientos y sus métodos a la evolución científica y pedagógica. La experiencia que se expone en este trabajo ha sido desarrollada en el seno del Centro del Profesorado Cuevas-Olula, de Almería, por lo que las referencias, fundamentalmente las normativas, se refieren a la Comunidad Autónoma Andaluza.

En la actualidad, el Decreto 110/2003 regula el Sistema Andaluz de Formación Permanente del Profesorado y la Orden de 9 de junio de 2003 aprueba el II Plan Andaluz de Formación Permanente del Profesorado. Los Centros de Profesorado (CEP) -así se denominan en Andalucía- son los encargados de articular este Plan de Formación. Estos centros son considerados como plataformas estables para la formación, la innovación y el intercambio de información pedagógica y para facilitar la formación de equipos de estudio y trabajo. Su institucionalización como espacios de encuentro y debate supone una apuesta por la organización de la formación ante los retos y cambios de los sistemas educativos, así como el desarrollo de distintas modalidades de formación generadas en y para los propios centros docentes.

En cualquier caso, la Ley 17/2007, de 10 de diciembre, de Educación de Andalucía (LEA), en su Sección 3 se refiere a la formación, haciendo expresa referencia en su $\operatorname{art}^{\circ} 18$ a la Formación Permanente del Profesorado y en su art $^{\circ} 20$ al Sistema Andaluz de Formación Permanente, organizado por la red de Centros del Profesorado (CEP).

En cuanto al planteamiento de las actividades formativas por parte de los CEP, es interesante destacar, por un lado, las diferentes estrategias que se desarrollan año a año para recabar una información lo más completa y actualizada posible, referente a las demandas formativas del profesorado de su ámbito de actuación; y, por otro lado, los asesores y asesoras encargados de elaborar el Plan de Actuación de cada CEP deben hacer lo posible por estar a la vanguardia de las temáticas de las que son responsables directos. En este sentido, la actualización científica y didáctica constituye una de las líneas de actuación que propone la Consejería de Educación de Andalucía (Granero, 2009), y la importancia de incluir en la programación de la Educación Física los contenidos de actividades físicas en el medio natural (AFMN) es cada día más obvia, y así llevamos dándonos cuenta desde hace décadas, al comprobar que cada día aumentan este tipo de prácticas, teniendo en cuenta que al alumnado no sólo le servirá como adquisición de aspectos motrices, sino también como recurso para organizar su tiempo libre y de ocio, como medio de disfrutar de la naturaleza.

De esta manera, debido a la demanda del profesorado de Educación Física en relación con contenidos relacionados con el medio natural, unido a la presencia de una instalación tan interesante y completa como el Canal de Remo de Cuevas del Almanzora (Almería), construido con motivo de la celebración de los XV Juegos Mediterráneos 2005, celebrados en Almería, supone que se demande especialmente el piragüismo entre las actividades formativas. Teniendo en cuenta lo que ello puede suponer, tanto la referida instalación como la promoción entre el profesorado de Educación Física, para el alumnado, en el Plan de Actuación del CEP Cuevas-Olula para el curso 2006/2007 se incardina esta actividad formativa que tiene como objetivo la actualización de contenidos del bloque de actividad física en el medio natural, como recoge el R.D. 1631/2006, concretados en el piragüismo, persiguiendo, en este caso, además, la promoción y utilización con carácter docente del citado Canal de Remo. 


\section{2.- METODOLOGÍA}

El objetivo de este trabajo es analizar una actividad de formación permanente del profesorado de Educación Física en torno a la innovación de los contenidos en el aula a través de actividades como el piragüismo, mediante la opinión de los participantes en la misma. Asimismo, se plantean, a partir del trabajo y conclusiones extraídas, unas perspectivas futuras de trabajo con el profesorado de primaria y secundaria para continuar y consolidar conocimientos en relación con los contenidos trabajados.

\section{Muestra}

Para este apartado se han utilizado los datos aportados por los participantes en el curso de formación permanente del profesorado. La muestra está compuesta por los 30 participantes, siendo el 40\%, docentes de la etapa de primaria y $60 \%$ de la etapa de secundaria (tabla 1 ).

Tabla 1.- Datos de la muestra

\begin{tabular}{lcccc}
\hline & Etapa & \multicolumn{2}{c}{ Edad } & Interinos \\
\cline { 2 - 5 } & $\%$ & $M$ & $S D$ & $\%$ \\
\hline Primaria & 40 & 33.3 & 2.1 & 29 \\
Secundaria & 60 & 35.7 & 2.6 & 71 \\
\hline
\end{tabular}

\section{Instrumento de evaluación}

El instrumento utilizado para la recopilación de la información ha sido el Cuestionario de Evaluación de Actividades Formativas (CEAF), cuyas preguntas hacen referencia a diferentes variables en relación a la calidad de la actividad desarrollada, del profesorado y de los contenidos trabajados, tales como: la duración, novedad de los contenidos, aplicabilidad, comprensión o actuación de los asistentes. Se trata de un instrumento utilizado y validado por parte del CEP para la evaluación de las distintas actividades formativas que desarrolla. El cuestionario, adaptado a la actividad realizada fue entregado al final de la misma y rellenado de forma autoadministrada por todo el profesorado participante.

Tabla 2.- Esquema del diseño de la actividad

\begin{tabular}{ll}
\hline & Despertar el interés hacia la práctica del piragüismo como medio de desarrollo \\
& personal, de relación con los demás y conocimiento y respeto del entorno \\
& natural. \\
& Proporcionar al nuevo palista los conocimientos y dominios básicos para \\
& buscar su autosuficiencia en la navegación por aguas tranquilas próximas a la \\
orilla. & Adquirir la formación básica sobre embarcaciones, sus diferentes usos y las \\
& posibilidades pedagógicas de cada tipo de embarcación, según la persona y el \\
& medio en el que la vamos a utilizar. \\
& Formar al profesorado para que sea capaz de llevar al alumnado hacia el \\
& conocimiento del piragüismo y a la primera toma de contacto de la actividad. \\
\hline & Generalidades del piragüismo \\
& El material \\
& Metodología del piragüismo \\
& Técnicas y maniobras fundamentales \\
& Planificación de la iniciación al piragüismo \\
& Seguridad \\
Didáctica aplicada
\end{tabular}




\section{Análisis estadístico}

Una vez rellenados los cuestionarios, se ha procedido a realizar la codificación y tabulación del mismo. Posteriormente, el análisis estadístico se ha realizado a través del paquete SPSS v.17, llevando a cabo un análisis descriptivo de las frecuencias.

\section{Diseño de la propuesta práctica}

La actividad formativa diseñada, cuyos objetivos y contenidos se pueden observar en la tabla 2, se plantea con la pretensión de que los docentes adquieran recursos e ideas suficientes para que sean capaces de crear sus propias propuestas en un entorno de costa y con fácil acceso a las playas. Esto puede suponer el planteamiento de sesiones innovadoras y cada vez más motivantes, a la vez que se pueden plantear objetivos interrelacionados con otras áreas del currículum. Asimismo, se trata de acercar el entorno a las aulas y a la inversa, de tal manera que los recursos de los que dispone el lugar en el que se desarrolla la actividad y que es ámbito de actuación del CEP, se pongan a disposición del alumnado a través de la formación del profesorado.

\section{Procedimiento y desarrollo}

Respecto al desarrollo de la actividad, reseñar que se ha pretendido fuera lo más enriquecedora posible, por lo que se plantearon sesiones variadas, tanto en contenidos, como en el lugar de desarrollo, de tal manera que cambiaran y combinaran las características del medio acuático.

Como se ha indicado anteriormente, se realizaron las pertinentes gestiones para desarrollar algunas sesiones en el referido Canal de Remo, pero el mismo no se hallaba en condiciones de albergar actividad alguna, provocado por el desuso inmediatamente posterior a la celebración de los Juegos Mediterráneos debido a la falta de coordinación de las distintas administraciones implicadas. Mientras ese acuerdo llegaba o no, el curso se puso en marcha, buscando alternativas a la lámina de agua tranquilas.

Para el desarrollo de los distintos contenidos y la consecución de los objetivos, tras las sesiones teóricas se realizó una fase de prácticas de seis sesiones (18 h.) en el Puerto de la Balsica de Villaricos, Cuevas del Almanzora (Almería), contando con la previa autorización de la Autoridad Portuaria de Garrucha, de la cual depende el mismo. Este pequeño puerto del levante almeriense puerto si tiene tráfico de embarcaciones y ofrece la posibilidad de trabajar el piragüismo en distintos niveles y con diferentes dificultades: lámina de aguas tranquilas, la playa anexa al puerto -ideal de cara al aprendizaje y donde el oleaje interviene en distintas proporciones-, y travesías a lo largo de la costa. De esta manera se pudieron trabajar ejercicios básicos de entrada, salida, auto-rescate, remolque y vaciado, así como maniobras de conducción, de propulsión, de equilibrio y rescates, esquimotajes, rutas y travesías; asimismo se trabajaron las distintas aplicaciones didácticas de los diferentes contenidos (figura 1 y 2).

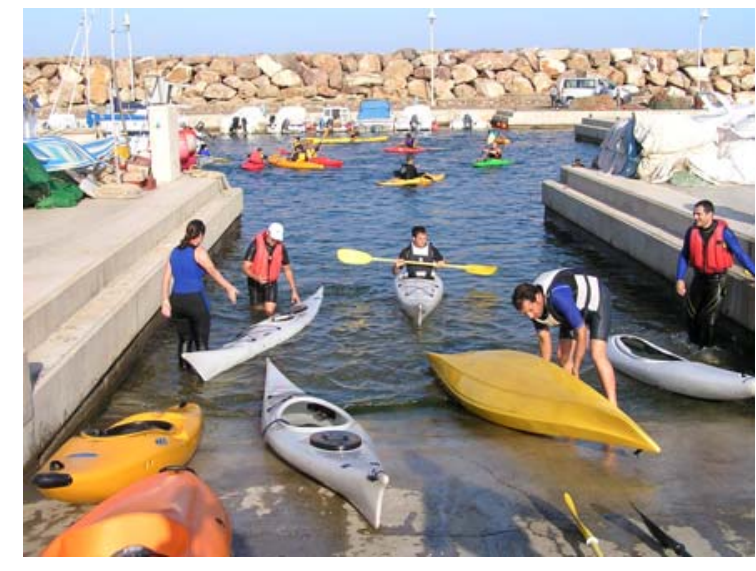

Figura 1. Prácticas en el Puerto de la Balsica de Villaricos

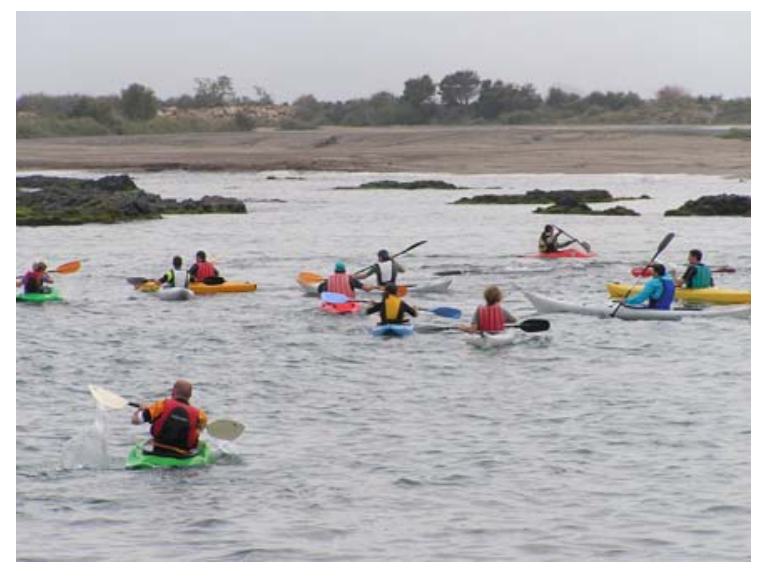

Figura 2. Prácticas en la playa 
Es interesante indicar que fueron treinta los profesores y profesoras de primaria y secundaria participantes como alumnado de esta actividad formativa y que para el desarrollo de las sesiones prácticas, en todo momento, dos de los tres profesores encargados de impartir el curso estuvieron en el agua con el alumnado, lo cual posibilitó, no sólo incrementar la seguridad y confianza de los participantes, sino trabajar por distintos niveles las diferentes secuencias de aprendizaje de los ejercicios propuestos.

Finalmente, dos sesiones (8 horas) en río servirían para completar la experiencia: práctica y ejecución de maniobras básica en el río, aplicando diferentes técnicas de palada y práctica de eslalon, en el Club Piragüismo de

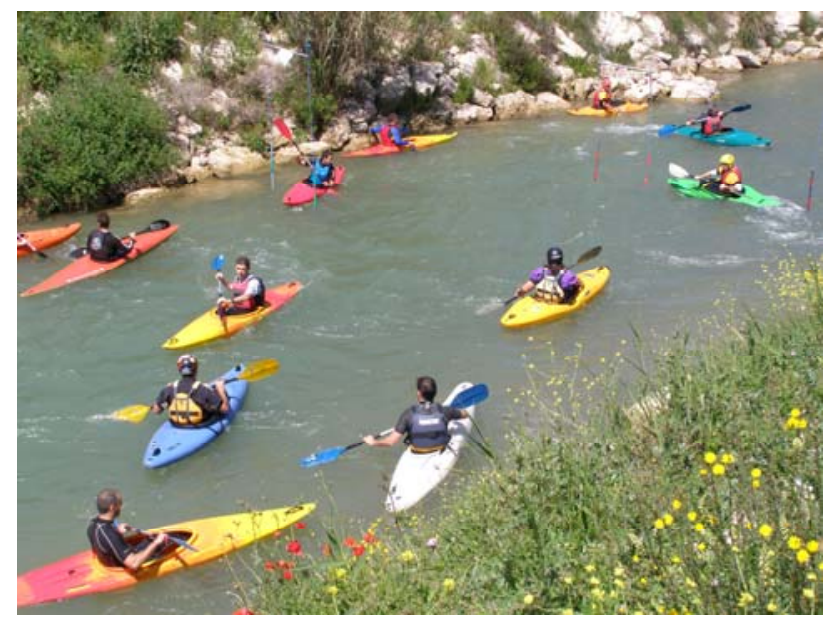

Figura 3. Prácticas en el Club de Piragüismo de Calasparra. Río Segura

Calasparra (Murcia) (figura 3), y un descenso por el río Segura (Murcia) (figura 4), de cara a conocer y complementar el aprendizaje en dos medios tan diferentes. Reseñar que se ha trabajado con diferentes tipos de embarcaciones para un aprendizaje más enriquecedor; distintos tipos de kayak de aguas bravas y surf, kayak de mar de distintos tamaños y kayak auto-vaciables (muy seguros y de gran confianza para los menos atrevidos).

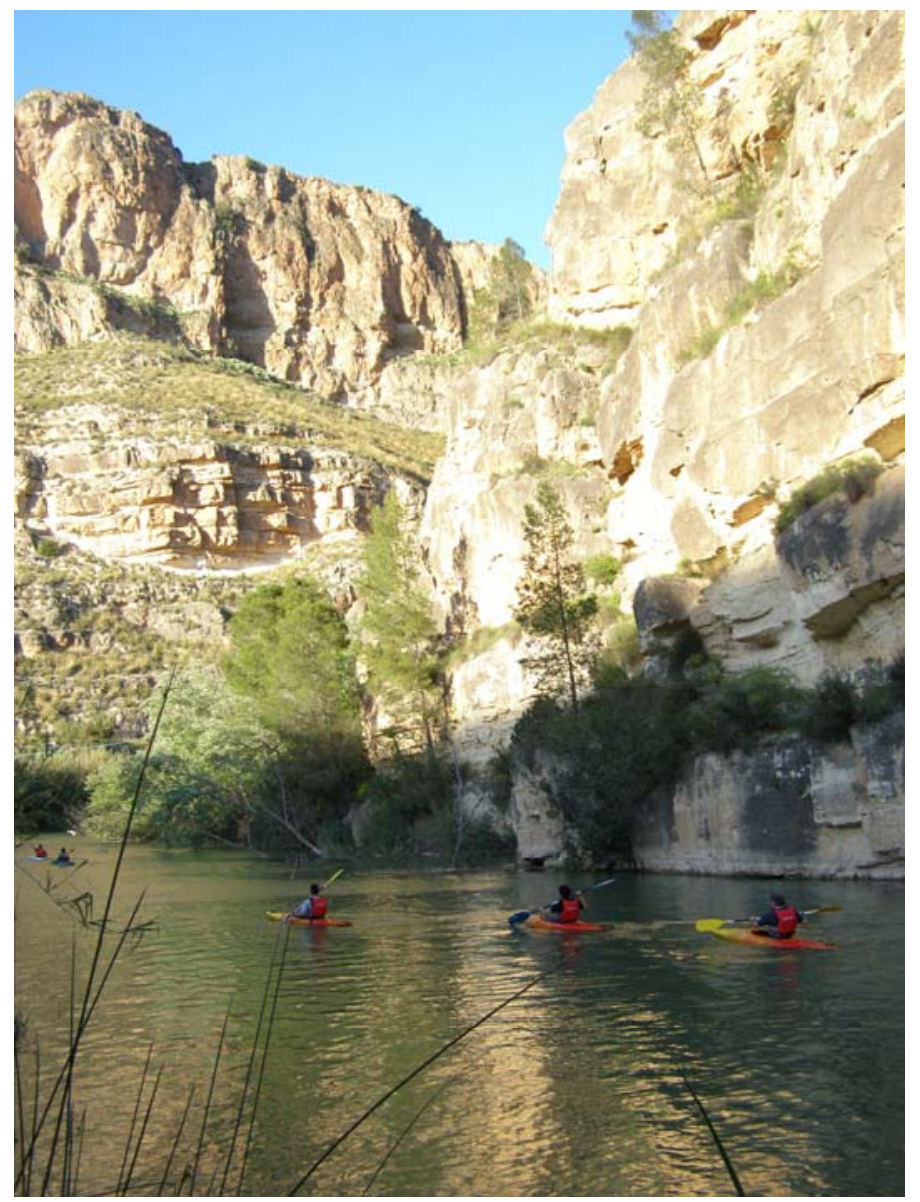

Figura 4. Descenso por el río Segura (Murcia) 


\section{3.- VALORACIÓN Y CONCLUSIONES}

A los 30 participantes (40\% de primaria y 60\% de secundaria) en esta actividad formativa se les pasó el cuestionario de evaluación de curso del Centro del Profesorado Cuevas-Olula, con cuestiones referentes a la calidad de la actividad, entorno, contenidos, etc.

En primer lugar, apuntar que para la mayoría, desde el punto de vista teórico, se trata contenidos novedosos (86\%), pero también interesantes (79\%).

Un aspecto que resulta de gran interés en el planteamiento de cursos de formación del profesorado es la posible aplicación práctica de los mismos en el aula, con posterioridad a la actividad formativa (Granero, 2009). Es decir, que tenga consecuencias positivas sobre el alumnado de primaria y secundaria. En este sentido, resalta el dato de que ocho de cada diez consideren aplicables los contenidos desde el punto de vista práctico, como se puede observar en la figura 5.

Las instalaciones y el entorno en el que se ha desarrollado la actividad han sido valoradas muy positivamente (figura 7) por el total de los participantes, prácticamente, por lo que se ha de resaltar especialmente el valioso complemento que ha supuesto navegar en el mar y en el río Segura, experiencia que completa un círculo muy importante en cuanto al aprendizaje y posterior enseñanza del piragüismo, ya

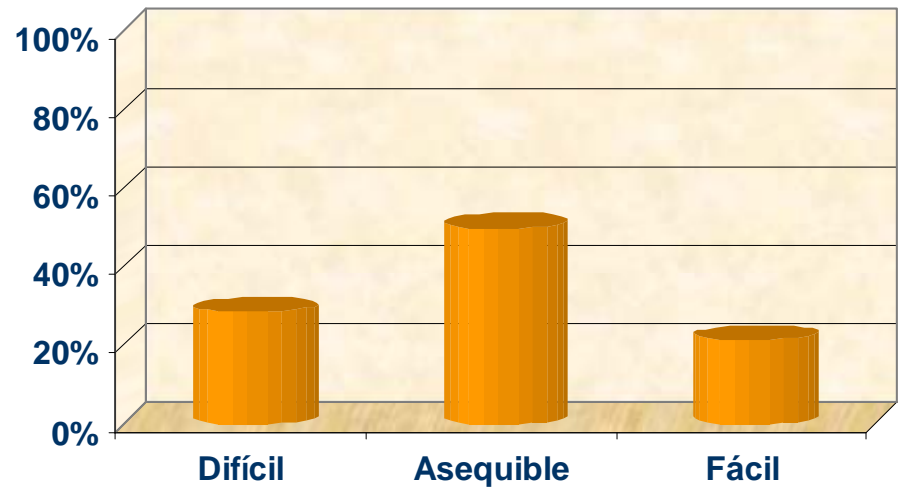

Figura 5. Posibilidad de aplicación práctica.

que son medios, que aunque pudiera creerse lo contrario, son totalmente distintos y que hay que conocer y experimentar para no cometer errores de seguridad y técnicos que podrían ser fatales en el desarrollo de una unidad didáctica con el alumnado.

Otro aspecto que preocupa en las actividades de formación del profesorado es la implicación y participación activa del mismo. Por ello se les pregunta para que hagan una valoración en relación a este aspecto del resto de participantes. En éste curso también se puede destacar y llamar la atención sobre el comportamiento, como se puede comprobar en la figura 6, del alto grado de implicación y participación del alumnado (93\%), según manifiestan haber percibido respecto a los propios compañeros.

Figura 6. Actuación de los asistentes, percibida por los propios participantes

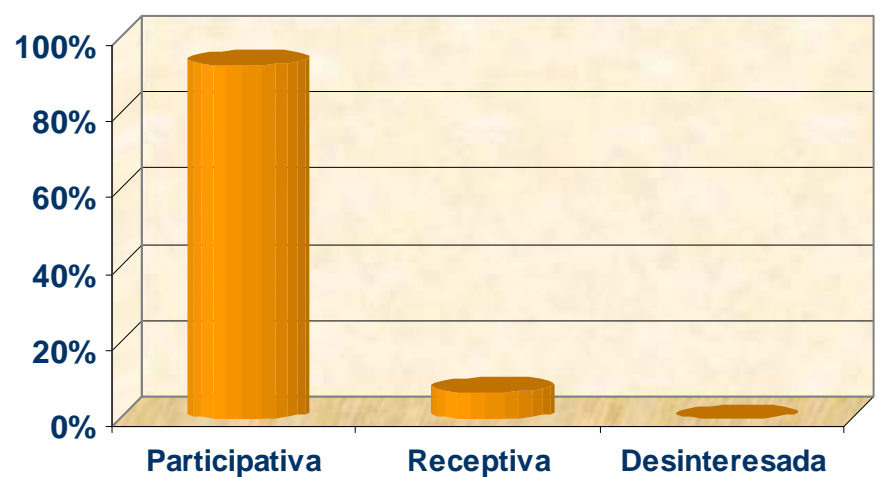


Finalmente, la valoración de la actividad no puede ser más positiva, si se observan la figura 7 , donde se exponen los datos respecto a los diferentes apartados del curso, valorado como muy bueno a nivel global, y no reflejando ninguno de los asistentes a la actividad, consideración negativa alguna.

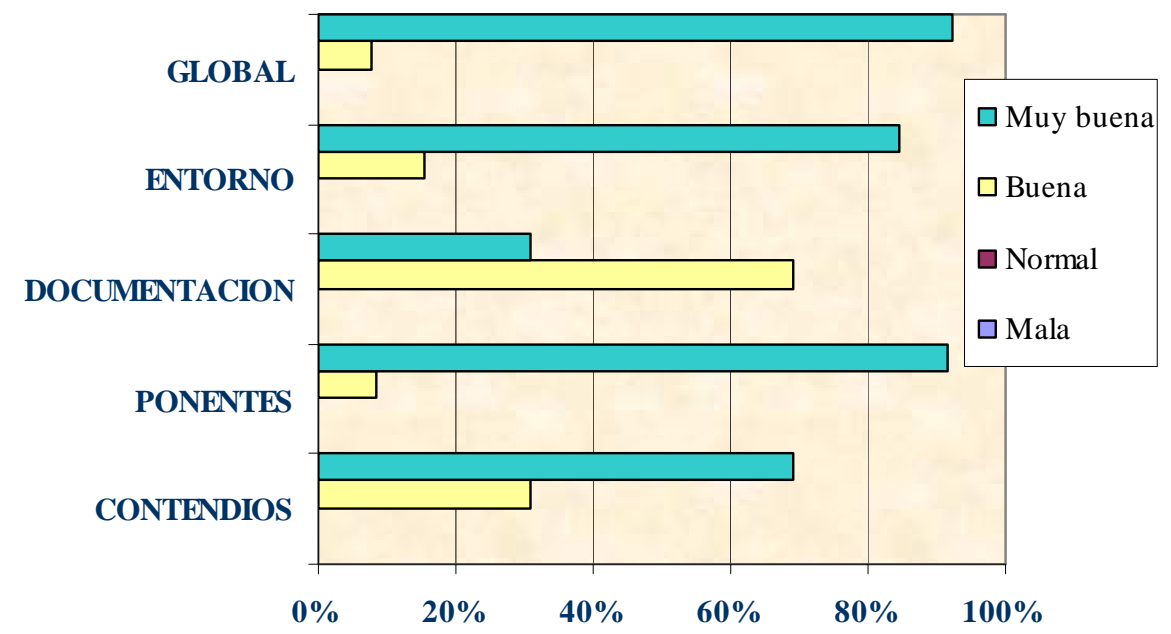

Figura 7. Valoración de distintos aspectos de la actividad

Dado lo expuesto, podemos afirmar que se han alcanzado los objetivos que se pretendían en esta actividad, lo cuales se plantearon a tres niveles.

En un primer lugar, conseguir que todo el profesorado que ha realizado el curso se adaptase a un medio que no siempre domina -el agua-, con un elemento con el que la mayoría no había tenido ninguna experiencia hasta ahora, la piragua. De esta manera se ha pretendido adquirir una seguridad personal por parte del profesorado participante -en este caso el alumnado-, traducido en confianza, para dar el paso de desarrollar con los alumnos y alumnas de los centros educativos actividades en el medio natural y más concretamente actividades en el medio acuático.

En un segundo nivel, se pretendía una formación básica sobre embarcaciones, sus diferentes usos y las posibilidades pedagógicas de cada tipo de embarcación, según la persona y el medio en el que la vamos a utilizar. Asimismo, también unos aspectos técnicos básicos y fundamentos amplios sobre seguridad tanto pasiva como activa.

La consecución de este objetivo supone la formación de profesores y profesoras con una formación adecuada para llevar a sus alumnos y alumnas a distintas actividades en el medio natural con las múltiples empresas que proliferan en la actualidad y que facilitan estas salidas, siendo capaces de valorar el trabajo de los monitores y empresas en su labor; sobre todo en cuanto a normas de seguridad y la metodología de enseñanza aprendizaje.

Como tercer nivel se debe de valorar si se incorpora la enseñanza de la piragua dentro de las clases de Educación Física, cuestión que se debe estimar en sucesivos años. Pero ya se dispone de una base para poder adaptar nuevos modelos de deportes practicados en el medio natural a las características específicas del trabajo diario de clase en Educación Física. También se encuentran cada vez más experiencias de estas característica entre la literatura de esta área, disponiendo de diferentes planteamientos didácticos llevados a la práctica y que pueden servir de orientación al profesorado (López y Granero, 2008a, 2008b).

Respecto a esta última consideración, apuntar que uno de los objetivos del CEP Cuevas-Olula al plantear este curso formativo de cara al profesorado iba en esta línea, en tanto en cuanto se abriera el Canal de Remo y la línea escolar de explotación del mismo suponga que el alumnado de primaria y secundaria tenga acceso a experiencias enriquecedoras relacionada con la piragua, canoas, etc.

Según consideran los propios asistentes, esta actividad pone de manifiesto las carencias que sobre los contenidos más innovadores de este bloque de contenidos tienen los docentes de Educación Física (Boned et al., 2002; Sáez, 2008). En este sentido, en el análisis aportado por Granero-Gallegos, 
Baena-Extremera y Martínez-Molina (2010), acerca de los contenidos desarrollados mediante las actividades en el medio natural de las clases de Educación Física en los cuatro cursos de la secundaria obligatoria, se expone claramente que los contenidos más trabajados en las aulas son los de orientación, senderismo y juegos; sin embargo, prácticamente no se trabajan contenidos relacionados con las actividades acuáticas. Es importante resaltar, en relación a lo expuesto, y como indican Granero-Gallegos y Baena-Extremera (2011), que el profesorado demanda formación sobre unos contenidos sobre los que apenas recibieron formados en la universidad. Por ello, adquieren gran importancia los planteamientos de formación permanente en estos tipos de contenidos.

Asimismo, no solo se encuentran cada vez más experiencias de estas características entre la literatura de esta área, disponiendo de diferentes planteamientos didácticos llevados a la práctica y que pueden servir de orientación al profesorado (Gass y Williamson, 1995; Breivik, 1996; Hattie et al., 1997; Attarian, 1999; Sibthorp, 2001; Baena, Calvo y Martínez, 2009; Baena y Granero, 2009; Granero et al., 2009; entre otros), sino que, además, cada vez más investigaciones se centran en el análisis que la repercusión del trabajo de estos contenidos tiene en el alumnado, tanto en secundaria (Baena-Extremera, Granero-Gallegos y Ortiz-.Camacho), como a nivel universitario (BaenaExtremera y Granero-Gallegos, in press).

Esta puede ser una manera para llevar realmente a las aulas de primaria y secundaria, partiendo de la formación permanente, contenidos innovadores y en línea con los intereses de la actual juventud, quienes demandan actividades con un mayor nivel de desafío y variedad de actividades de aventura al aire libre (Flintoff y Scraton, 2001; Rikard y Banville, 2006). Como han señalado diversos autores (Jaén y Banet, 2003; Granero-Gallegos y Baena-Extremera, 2011), la formación inicial del profesorado dista mucho de ser los adecuados para que los futuros docentes puedan afrontar con ciertas garantías de éxito la difícil empresa de enseñar. Pero además, al tratarse de contenidos innovadores, no son tratados en la formación inicial del docente y su inclusión hoy por hoy en el currículum adquiere gran importancia.

Por último, resaltar que la celebración de esta actividad formativa ha tenido una repercusión muy positiva y ha supuesto un incentivo más para que a nivel municipal se tomaran las iniciativas oportunas y el Canal de Remo se pusiera en funcionamiento. Los primeros docentes ya tienen reservas para la visita y práctica con su alumnado en esta instalación, en la actualidad.

\section{4.- REFERENCIAS BIBLIOGRAFICAS}

Attarian, A. (1999). Artificial climbing environments. En J.C. Miles, y S. Priest (Eds.), Adventure Programming (pp. 341-346). State College, PA: Venture Pub.

Baena Extremera, A., y Granero-Gallegos, A. (in press). Estudio cuasi-experimental de un programa de supervivencia en el medio natural. Revista Internacional de Medicina y Ciencias de la Actividad Física y el Deporte.

Baena Extremera, A., Granero-Gallegos, A., y Ortiz Camacho, M.M. (2012) Quasi-experimental Study of the Effect of an Adventure Education Programme on Classroom Satisfaction, Physical Self-Concept and Social Goals in Physical Education. Psychologica Belgica, 52(4), 369-386.

Baena, A., y Granero, A. (2009). Deportes de aventura indoor, enseñanza de la espeleología y los institutos de educación secundaria. Tándem. Didáctica de la Educación Física, 30, 47-60.

Baena, A., Calvo, J.F., y Martínez, M. (2009). Una experiencia didáctica en metodología integrada: cinco estilos de enseñanza a través de la bicicleta de montaña, patines y monopatines en E.S.O. [en línea]. Espiral. Cuadernos del Profesorado, 2(3), 41-48. Disponible en: http://www.cepcuevasolula.es/espiral. [Consulta: 7 de mayo].

Boned, C., Mayorga, J.I., Rivero, A., y Rodríguez, G. (2002). Preferencias formativas y profesionales de los alumnos de la Facultad de Ciencias de la Actividad Física de la UE-CEES: el caso de la gestión deportiva. Revista Kronos, 1, 25-33.

Breivik, G. (1996). Personality, Sensation Seeking and Risk Taking among Everest Climbers. International Journal Sport Psicology, 27, 308-320.

Centro de Profesorado de Cuevas Olula (2007). Plan de Actuación 2006-2007. Disponible en: http://www.cepcuevasolula.es. Consulta [2007, 23 de noviembre]. 
Decreto 110/2003, de 22 de abril, por el que se regula el Sistema Andaluz de Formación Permanente del Profesorado. BOJA núm. 78, de 25 de abril de 2003.

Flintoff, A., y Scraton, S. (2001). Stepping into Active Leisure? Young Women's Perceptions of Active Lifestyles and their Experiences of School Physical Education. Sport, Education and Society, 6(1), 521.

Gass, M.A., y Williamson, J. (1995). Accreditation for adventure programs. Journal of Physical Education, Recreation and Dance, 66(1), 22-27.

Granero, A. (2009). La formación permanente del profesorado no universitario. En J.M. Martínez y A. Codina (Eds.), La Formación Inicial del profesorado desde las Prácticas de Enseñanza. Retos y perspectivas para su mejora (pp. 103-116) [CD ROM]. Almería: Universidad de Almería.

Granero, A., López, J.L., Godoy, M., Núñez, J.J., Núñez, M., Baena, A., et al. (2009). La Educación Física a través de un currículum común en centros de secundaria [en línea]. Espiral. Cuadernos del Profesorado, 2(3), 55-65. Disponible en: http://www.cepcuevasolula.es/espiral. [Consulta: 2009, 6 de mayo].

Granero-Gallegos, A., Baena-Extremera, A., y Martínez-Molina, M. (2012). Contenidos desarrollados mediante las actividades en el medio natural de las clases de Educación Física en secundaria obligatoria. Ágora para la Educación Física y el Deporte, 12(3), 273-288.

Granero-Gallegos, A., y Baena-Extremera, A. (2011). Juegos y deportes de aventura en la formación permanente del profesorado. Revista Internacional de Medicina y Ciencias de la Actividad Física y el Deporte, 11(43), 531-547. Disponible en: http://cdeporte.rediris.es/revista/revista43/artjuegos224.htm

Hattie, J., Marsh, H.W., Neill, J.T., y Richards, G.E. (1997). Adventure education and Outward Bound: Out-ofclass experiences that make a lasting difference. Review of Educational Research, 67(1), 43-87.

Jaén, M., y Banet, E. (2003). Formación inicial de profesores de secundaria: dificultades para aprender a planificar y desarrollar las actividades de enseñanzas en las aulas de secundaria. Revista Interuniversitaria de Formación del Profesorado, 17(1), 51-78

Ley 17/2007, de 10 de diciembre, de Educación de Andalucía. BOJA núm. 252 de 26 de diciembre de 2007.

López J.L., y Granero, A. (2008a). 'Disfrutando del mar con la piragua'. Unidad Didáctica para Educación Física en la secundaria post-obligatoria. Lecturas Educación Física y Deportes, Revista Digital [en línea], 118. Buenos Aires. Disponible en: http://www.efdeportes.com [Consulta: 2009, 2 de febrero].

López J.L., y Granero, A. (2008b). Una experiencia de orientación en piragua desarrollada en el medio natural. Tándem. Didáctica de la Educación Física, 28, 107-118.

Orden de 9 de junio de 2003, por el que se aprueba el II Plan Andaluz de Formación Permanente del Profesorado. BOJA núm. 121, de 26 de junio de 2003.

Real Decreto 1631/2006, de 29 de diciembre, por el que se establecen las Enseñanzas Mínimas correspondientes a la ESO. BOE $\mathrm{n}^{\circ} 5$ de 5 de enero de 2007.

Rikard, G.L. y Banville, D. (2006). High school student attitudes about physical education. Sport, Education and Society, 11(4), 385-400.

Sáez, J. (2008). El diseño de las actividades en el medio natural en el nuevo currículum de la educación secundaria obligatoria a partir de la Ley Orgánica de Educación (LOE). Ágora para la Educación Física y el Deporte, 7-8, 99-124

Sibthorp, J. (2001). Measuring weather...and adventure education: Exploring the instruments of adventure education research. Journal of Experiential Education, 23(2), 99-1

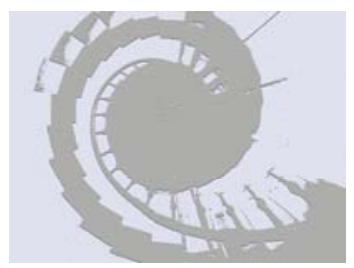

